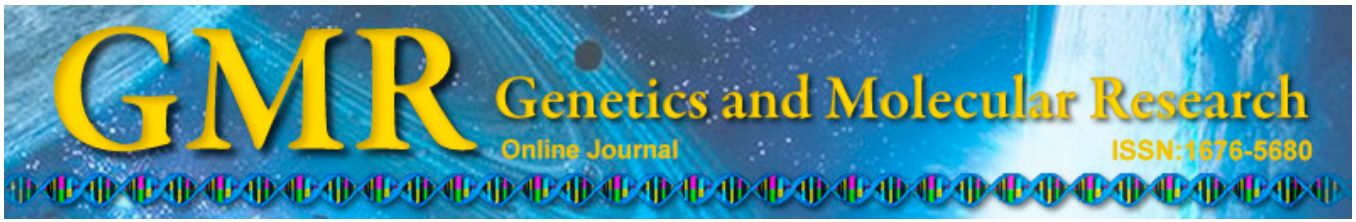

\title{
Clinical application of three-dimensional reconstruction and rapid prototyping technology of multislice spiral computed tomography angiography for the repair of ventricular septal defect of tetralogy of Fallot
}

\author{
X.J. Ma' , L. Tao ${ }^{2}$, X. Chen ${ }^{2}$, W. Li ${ }^{1}$, Z.Y. Peng ${ }^{1}$, Y. Chen ${ }^{1}$, J. Jin ${ }^{2}$, \\ X.L. Zhang ${ }^{1}$, Q.F. Xiong ${ }^{1}$, Z.L. Zhong ${ }^{1}$ and X.F. Chen ${ }^{1}$ \\ ${ }^{1}$ Department of Radiology, Wuhan Asia Heart Hospital, Wuhan, \\ Hubei Province, China \\ ${ }^{2}$ Department of Cardiac Surgery, Wuhan Asia Heart Hospital, Wuhan, \\ Hubei Province, China \\ Corresponding author: X. Chen \\ E-mail: xinChencn@163.com
}

Genet. Mol. Res. 14 (1): 1301-1309 (2015)

Received May 25, 2014

Accepted October 30, 2014

Published February 13, 2015

DOI http://dx.doi.org/10.4238/2015.February.13.9

\begin{abstract}
Three-dimensional (3D) reconstruction and rapid prototyping technology (RPT) of multislice spiral computed tomography angiography (CTA) was applied to prepare physical models of the heart and ventricular septal defects of tetralogy of Fallot (ToF) patients in order to explore their applications in the diagnosis and treatment of this complex heart disease. CTA data of $35 \mathrm{ToF}$ patients were collected to prepare 1:1 3D solid models using digital 3D reconstruction and RPT, and the resultant models were used intraoperatively as reference. The operations of all 35 patients were completed under the guidance of the 3D solid model, without difficulty. Intraoperative findings of the patients were consistent with the morphological and size changes of the
\end{abstract}


3D solid model, and no significant differences were found between the patches obtained from the 3D solid model and the actual intraoperative measurements $(t=0.83, \mathrm{P}=0.412)$. 3D reconstruction and RPT of multislice spiral CTA can accurately and intuitively reflect the anatomy of ventricular septal defects in ToF patients, providing the foundation for a solid model of the complex congenital heart.

Key words: Tetralogy of Fallot; Ventricular septal defect; $3 \mathrm{D}$ reconstruction; Rapid prototyping

\section{INTRODUCTION}

With the rapid development of cardiac surgical technologies and thoracoscopic cardiac surgery, anincreasing number of structural heartdiseases, including some complex congenitalheart diseases, arebeingidentifiedinearlystagesandarebeingtreatedwithradicalsurgery.Becauseanumber of complex structural deformities can be easily misdiagnosed, patients have high surgical risk, for example: the wrong choice of surgical approach leads to prolonged surgery; postoperative low cardiac output, arrhythmia, bleeding, infection, renal or multiple organ failure was significantly higher, and even increased mortality. Therefore, a detailed preoperative understanding of the complex anatomy and spatial relationships in patients with cardiovascular malformations is urgently desired by every cardiac surgeon (Kim et al., 2008a). Current two-dimensional (2D) imaging technology remains suboptimal for the anatomy and spatial relationships in structural complex heart diseases. RPT is a new digital prototyping technology based on the discrete accumulation-forming principle and the combination of multiple subjects, such as computeraided design, computer numerical control (CNC), laser, and new materials (Esses et al., 2011). Because of its productive agility, highly integrated manufacturing technology, and ability to produce a three-dimensional (3D) entity with any complex shape, RPT has been widely used in the medical field; however, only a few institutions have reported its use in the field of cardiovascular disease (Kim et al., 2008b). In this study, by constructing 3D solid models of the heart and ventricular septal defects in ToF patients, we explored the applications of 3D reconstruction and RPT of multislice spiral CT angiography (CTA) in cardiovascular diseases.

\section{MATERIAL AND METHODS}

\section{General information}

Data were collected from 35 ToF patients (19 males and 16 females) who had been echocardiographically diagnosed and surgically treated at our hospital between May 2012 and June 2013. Patient age range was 2-36 years, with a mean age of 8.2 years. Of the total patients, 24 had simple ToF, 7 cases of patients accompanying atrial septal defect (ASD), and 4 cases of patients accompanying patent ductus arteriosus (PDA). This study was reviewed and approved by the ethics committee of Wuhan Asia Heart Hospital. The legal guardians of all patients were informed and signed the informed consent. All children who were unable to cooperate with the procedure were orally administrated chloral hydrate $(50-100 \mathrm{mg} / \mathrm{kg})$ or received intravenous anesthesia (propofol, 2.0-2.5 mg/kg) for 4-5 minutes. All patients were observed in the CT room for half an hour before they were sent back to the ward for continuous observation. 


\section{CTA scan parameters}

All patients underwent scanning with the Siemens Flash dual-source CT (Somatom Definition; Siemens, Forchheim, Germany). Retrospective electrocardiac gating technology was applied, with the automatic trigger threshold at $100 \mathrm{HU}$ and the low-dose controlling program. The main parameters of dual-source CT scanning were: scan collimator thickness $2 \mathrm{x}$ $32 \times 0.6 \mathrm{~mm}$, tube rotation time: $270 \mathrm{~ms} /$ week, tube voltage $80-100 \mathrm{KV}$, tube current 100-200 $\mathrm{mA}$, and pitch 0.5 . Image reconstruction was performed at the end systolic $(45 \%)$ and diastolic $(75 \%)$ periods, respectively, with a reconstruction thickness of $0.625 \mathrm{~mm}$. The overlapping reconstruction was then performed, and the images were transferred into the Philips EBW V4.5 workstation (Philips, Amsterdam, Holland) for analysis. Post-processing methods included multiplanar reconstruction, maximum intensity pixel, and volume rendering. A non-ionic contrast agent (Omnipaque 320, GE Healthcare, USA) was used, and a $20 \mathrm{G}$ venous catheter was implanted in the patients' arm or leg. Using a dual barrel syringe (Stellant; Medrad, Pittsburgh, PA.), a dose of $10-20 \mathrm{~mL}(1-2 \mathrm{~mL} / \mathrm{kg})$ of contrast was injected at a flow rate of $0.5 \mathrm{~mL} / \mathrm{s}$, accompanied by an appropriate saline bolus injection at the same speed. The trigger interest zone was set in the descending aorta, and the scanning range started from the neck root and ended at the diaphragm level.

\section{Production and pre-preparation of the 3-D solid model}

Original patient data obtained at end diastole (75\%) were imported into the CompCardiac post-processing software package of Philips EBW (V4.5) workstation for digital $3 \mathrm{D}$ reconstruction of the patients' heart shape, and via surgery, the right ventricular free wall was excised to obtain the ventricular septal defect model. The results were converted by the CNC, generating files in Standard Tessellation Language (STL) format. The STL data were then sent to the HRPS-IV for laser sintering (Wuhan Binhu Mechanical \& Electrical Co., Ltd., Huazhong University of Science and Technology, Wuhan, China), using selective laser sintering technology and certain laser parameters to sinter the PSB powder into a 1:1 3-D solid model of the heart and ventricular septal defect. Using the combined 3-D solid model and other imaging data, we communicated our findings to the patients and their families and discussed surgical planning. A simulative surgical defect was performed on the solid model and later compared with the actual data measured intraoperatively. After packing, cryogenic ethylene oxide sterilization was performed for the model, and in the operating room, intraoperative real-time observation of the spatial relationships of the ventricular septal defect, tricuspid valve, and left ventricular outflow tract were performed on the model.

\section{Intraoperative application of the 3-D solid model}

All 35 patients with ToF underwent radical surgery via the transthoracic approach. After general anesthesia induction and nasal endotracheal intubation, each patient was placed in the supine position, maintaining head traction. Routine cleaning of the neck, chest, and abdomen was then performed, followed by iodine disinfection of the chest, neck, and both axillary sides. A sternal dilator was used to distract the split sternum, and the pericardium was cut to reveal the heart. The right ventricular outflow 
tract was then longitudinally incised $2-4 \mathrm{~cm}$ to reveal the ventricular septal defect; thus, the size of the ventricular septal defect could be measured and compared with the data obtained from the 3-D solid model. The spatial structure of the solid model was used as a reference to repair the ventricular septal defect. For patients with other accompanying cardiac malformations, the surgical program for the corresponding deformity was also performed.

\section{Postoperative processing}

Each patient was monitored in the intensive care unit (ICU) for 24-48 h after surgery and was evaluated with an echocardiogram to review changes in cardiac function, tricuspid valve motor function, and morphologies of the left and right ventricular outflow tracts. The tracheal cannula was maintained for 6-12 h postoperatively, and patients were hospitalized for 1-week observation after the surgery. Intravenous antibiotics were administered for 5-7 days postoperatively.

\section{Statistical analysis}

Data are reported as (means \pm SD), and the SPSS 19.0 statistical software was used for the $t$-test of shape and size of the ventricular septal defect before and after surgery, with $\mathrm{P}$ $<0.05$ considered to be statistically significant.

\section{RESULTS}

For all 35 patients, solid models of the heart shape and ventricular septal defect were created using 3D reconstruction and RPT (Figures 1-4). Shapes and sizes of the ventricular septal defects, as well as the structural changes of lesions found intraoperatively, were consistent with those revealed by the preoperative 3-D solid models. No significant differences were found between the data measured by the preoperative 3-D solid models and the actual intraoperative measurements $(t=0.83, \mathrm{P}=0.412>0.05)$ (Table 1$)$. The length of hospitalization for the 35 patients was 10-21 days, with an average of 11.5 days. Patients were followed up for 6-18 months, with an average of 8.5 months. All surgeries were completed according to the preoperative surgical planning and were uneventful. No patient developed complications such as atrioventricular block or tricuspid valve dysfunction. The shapes of left and right outflow tracts were normal, with no evidence of outflow obstruction. The ventricular septal defects were stitched well, with a satisfactory patch shape and size.

$\begin{aligned} & \text { Table 1. Comparison of ventricular septal defect between the 3-D solid model and the intraoperative actual } \\
& \text { measurement. }\end{aligned}$
\begin{tabular}{lllr} 
& Mean & N & SD \\
\hline Model measurement $(\mathrm{mm})$ & 14.98 & 35 & 1.91 \\
Surgical measurement $(\mathrm{mm})$ & 15.11 & 35 & 2.06 \\
\hline
\end{tabular}




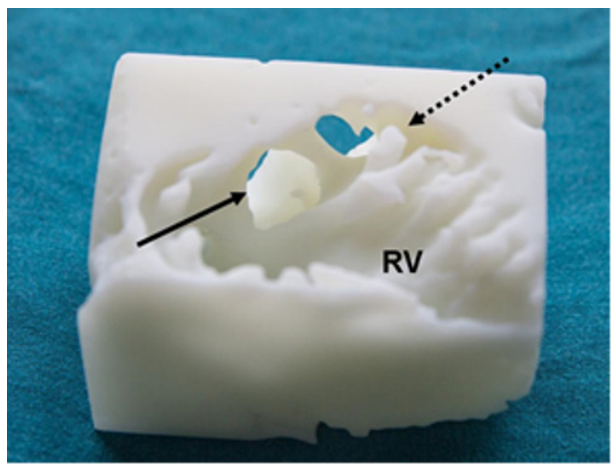

Figure 1. Male, 2 years old, exhibited the cyanosis since his childhood. The cross-sectional view of right ventricule, the ventricular septal defect located on the peri-membranous (solid arrow). The right ventricular outflow tract located left to the ventricular septal defect (imaginary arrow). RV: right ventricle.

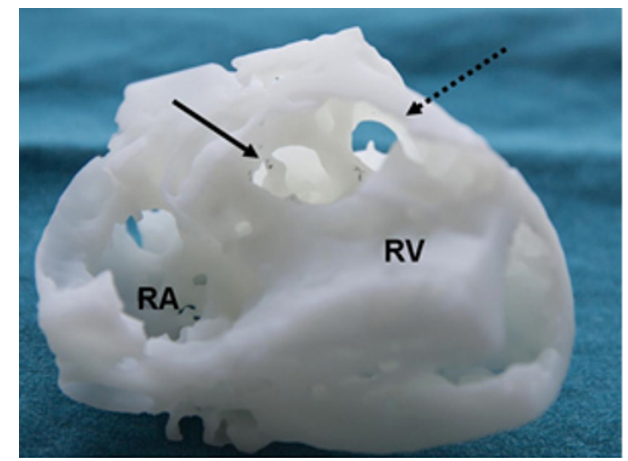

Figure 2. Male, 3 years old, exhibited the cyanosis since his childhood. The cross-sectional view of right ventricule, the ventricular septal defect located on the peri-membranous (solid arrow). The right ventricular outflow tract located left to the ventricular septal defect (imaginary arrow). RV: right ventricle, RA: right atrium.

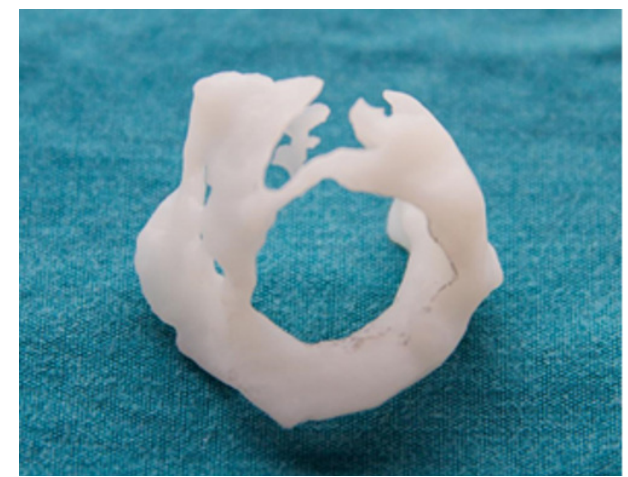

Figure 3. Male, 2.5 years old, exhibited the cyanosis since his childhood. 3-D printing figure of ventricular septal defect. 


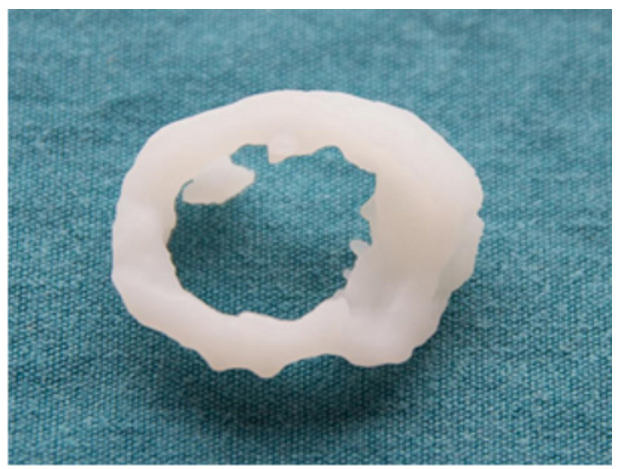

Figure 4. Female, 2 years old, exhibited the cyanosis since her childhood. 3-D printing figure of ventricular septal defect.

\section{DISCUSSION}

\section{Introduction of RPT}

RPT converts a 3-D computer complanate model into a physical solid model. This technology was originally applied in industrial manufacturing to design components and manufacture automobiles, aircraft, computers, and other products. Among industrial applications, RPT is mainly used to evaluate the feasibility of assembly of newly designed products prior to large-scale manufacturing. Medical RPT was first introduced in 1990 when original CT data were used to prepare a skull model that could display fully detailed internal anatomical structures (Mankovich et al., 1990). Since then, rapid advances in medical imaging technologies, computer hardware, and 3-D image processing software have promoted the development of medical RPT. Because of its high precision in model production and short production period, RPT is especially suitable for creating complex structural models. In recent years, it has been widely used in such clinical medical fields as dentistry, maxillofacial surgery, orthopedics, and orthopedic and prosthetic engineering, providing effective solutions and production methods (Heissler et al., 1998; Sailer et al., 1998; D’Urso et al., 1999; Winder and Bibb, 2005). It is also used in orthopedic surgery (Munjal et al., 2000; Minns et al., 2003), both in clinical medicine for surgical planning and in biomanufacturing. As an advanced technology, RPT could be used for model construction in a variety of fields and could be helpful in depicting peripheral vessels and the vasculature in the brain (Knox et al., 2005).

\section{Value of RPT in repair of the ventricular septal defect of ToF}

In recent years, because of the development of noninvasive diagnostic techniques such as CT, magnetic resonance imaging (MRI), and positron emission tomography, clinicians can easily obtain 2-D sectional images of relevant parts in patients and use a computerized digital 3-D reconstruction to perform associated measurements of lesions. Although digital 3 -D reconstruction can provide accurate anatomical information, the necessary equipment and the absence of the real-time information intraoperatively limit its use. In addition, a 2-D screen cannot visually present 3-D geometrical structures of virtual computerized models; thus, rare or complicated cardiac malformations can be difficult to observe on the screen, 
whereas a tangible solid model provides a much more intuitive way of understanding complex anatomical structures. The main goal of ToF radical surgery is to repair the ventricular septal defect. Because the defect is generally adjacent to the tricuspid valve and is surrounded by rich conductive tracts, damage to the tricuspid valve and surrounding conductive tracts must be avoided. An RPT 3-D solid model can clearly show the spatial relationship between the ventricular septal defect and the tricuspid valve as well as the morphology of the right ventricular outflow tract. High-precision three-dimensional reconstruction image obtained by modern multifunctional imaging technology including multi-slice spiral CT (MSCT), magnetic resonance imaging (MRI) and three-dimensional echocardiography, that combined with rapid prototyping technology has begun to expand into medical applications, it may directly affect the patient's treatment. (Binder et al., 2000; Pentecost et al., 1999, 2001; Gilon et al., 2002; Ngan et al., 2006; Noecker et al., 2006; Spence et al., 2006; Schievano et al., 2007; Sodian et al., 2007). Cardiovascular RPT could intraoperatively assist with the spatial location of cardiovascular malformations as well as their repair. It has been shown that in addition to enabling clinicians to further understand complex anatomical structures, this technology can significantly improve the diagnostic accuracy of disease; thus, it would be helpful in preoperative planning, enhancing the teaching effectiveness of medical students and clinicians, and facilitating communication with patients and their families (Petzold et al., 1999).

Based on the original imaging data of 10 CTA patients, Kim et al. (2008b) applied stereolithography apparatus technology to produce corresponding solid anatomical models of cardiac malformations, including atrial septal defect, ventricular septal defect, patent ductus arteriosus, and aortoarteritis, and used the models to simulate the planned surgical closures. The occluders subsequently selected according to the solid model simulation proven to be very suitable for the surgeries. The application of solid models could increase the safety of cardiac surgery, reduce operative time, improve exchanges within the surgical team, and facilitate better communication with patients and their families, thus minimizing misunderstandings and errors. The results of our study were consistent with the results above, and the postoperative satisfaction of our patients was high.

\section{Key technology of cardiac CTA 3-D reconstruction and RPT solid model production}

Heart three-dimensional imaging technology is different from orthopaedic and dental organizational structure with high density, heart three-dimensional imaging is not readily obtained ideal model in the process of reprocessing. In order to obtain a satisfactory cardiovascular solid model, an iodine-containing contrast agent must be intravenously injected, and each chamber of the heart must be filled with the appropriate concentration of the contrast agent, especially in the ventricles. Our experience was that the contrast concentration was 200-350 HU within the left ventricle and 150-250 HU within the right ventricle. Therefore, during the cardiac data collection process, the choice of triggering CT scanning was very important. In the author's institute, after the ulnar intravenous injection of contrast, the preset threshold-automatic triggering mode was used. The interest point should usually be set in the descending aorta, and the threshold value was set at $200 \mathrm{HU}$. In addition to accurate scanning, the post-3D-processing cutting is the key to ensuring a good solid model. Because the heart has a rich supply of blood vessels, the 3-D processing staff must be skilled in post-processing techniques and possess comprehensive knowledge of cardiovascular anatomy and pathology. Use of the advanced Philips EBW (V4.5) workstation and the Comp-Cardiac post-processing 
software package allowed the cardiac solid model to be easily obtained. The requirements of the operator in reproducing the cardiac ventricular septal defect are relatively high. A surgical approach is used to resect the right ventricular free wall; then, the ventricular septal defect is displayed by the right ventricular section, after which this part is carefully cut. Thus, the desired ventricular septal defect model is obtained.

\section{Significance of cardiac CTA 3-D reconstruction and RPT}

This preliminary study applied cardiac CTA 3-D reconstruction and RPT for the diagnosis of complex congenital cardiac malformations. The advantages and meaning of this approach are as follows: 1) The RPT solid model provides a reference for the diagnosis and treatment of complex congenital heart diseases, helps with the development and implementation of surgical programs, and provides intraoperative spatial position guidance for surgery. Radical surgery of complex congenital heart disease is precise with strict time constraints. If a 3-D solid model could be used as a basis for preoperative surgical planning for this complex surgery, it would have important clinical significance in reducing the surgical and perfusion time, thus contributing to successful surgery and postoperative recovery. 2) The RPT solid model can produce a 1:1 representation of cardiovascular malformations and can not only be used as a platform for training young doctors, but also add to the knowledge among experienced physicians. 3) Because this technique would be the best reference among physicians and nurses for communication with the patients and their families, the RPT solid model could reduce misunderstandings between doctors and patients. Thus, the intent and effect of surgery could be best implemented and understood.

\section{Limitations and outlook of this study}

Although this study showed satisfactory results, the total number of cases and types of diseases were limited. Further, the value of using cardiovascular CTA 3-D reconstruction and RPT in other cardiovascular diseases still needs more research for confirmation. The 2-D raw data of this study were collected through a multislice spiral CT. Therefore, patients received a small amount of radiation during the examination. By improving the scanning program and strengthening radiological protection, the patient radiation dose could be decreased to an acceptable low range (Chen et al., 2013).

In summary, this study used an RPT solid model to simulate the surgical repair of the ventricular septal defect of ToF, verified the feasibility of solid duplication of complex soft tissue structures, provided a reference for the extraction and solid model processing of 3-D data for other soft tissues, and explored a new direction for RPT in the medical field.

\section{ACKNOWLEDGMENTS}

Research supported by the Clinical Medicine Scientific Research Foundation of Wuhan City Health and Family Planning Commission. Hubei Province (\#WX12A07).

\section{REFERENCES}

Binder TM, Moertl D, Mundigler G, Rehak G, et al. (2000). Stereolithographic biomodeling to create tangible hard copies 
of cardiac structures from echocardiographic data: in vitro and in vivo validation. J. Am. Coll. Cardiol. 35: 230-237.

Chen X, Qu YJ, Peng ZY, Lu JG, et al. (2013). Diagnosis of congenital aortic arch anomalies in Chinese children by multidetector computed tomography angiography. J. Huazhong. Univ. Sci. Technolog. Med. Sci. 33: 447-451.

D’Urso PS, Barker TM, Earwaker WJ, Bruce LJ, et al. (1999). Stereolithographic biomodelling in craniomaxillofacial surgery: a prospective trial. J. Craniomaxillofac. Surg. 27: 30-37.

Esses SJ, Berman P, Bloom AI and Sosna J (2011). Clinical applications of physical 3D models derived from MDCT data and created by rapid prototyping. AJR Am. J. Roentgenol. 196(6):683-688.

Gilon D, Cape EG, Handschumacher MD, Song JK, et al. (2002). Effect of three-dimensional valve shape on the hemodynamics of aortic stenosis: three-dimensional echocardiographic stereolithography and patient studies. J. Am. Coll. Cardiol. 40: 1479-1486.

Heissler E, Fischer FS, Bolouri S, Lehmann T, et al. (1998). Custom-made cast titanium implants produced with CAD/ CAM for the reconstruction of cranium defects. Int. J. Oral. Maxillofac. Surg. 27: 334-338.

Kim MS, Hansgen AR and Carroll JD (2008a). Use of rapid prototyping in the care of patients with structural heart disease. Trends Cardiovasc. Med. 18: 210-216.

Kim MS, Hansgen AR, Wink O, Quaife RA, et al. (2008b). Rapid prototyping: a new tool in understanding and treating structural heart disease. Circulation 117: 2388-2394.

Knox K, Kerber CW, Singel SA, Bailey MJ, et al. (2005). Stereolithographic vascular replicas from CT scans: choosing treatment strategies, teaching, and research from live patient scan data. AJNR Am. J. Neuroradiol. 26: 1428-1431.

Mankovich NJ, Cheeseman AM and Stoker NG (1990). The display of three-dimensional anatomy with stereolithographic models. J. Digit. Imaging. 3: 200-203.

Minns RJ, Bibb R, Banks R and Sutton RA (2003). The use of a reconstructed three-dimensional solid model from CT to aid the surgical management of a total knee arthroplasty: a case study. Med. Eng. Phys. 25: 523-526.

Munjal S, Leopold SS, Kornreich D, Shott S, et al. (2000). CT-generated 3-dimensional models for complex acetabular reconstruction. J. Arthroplasty 15: 644-653.

Ngan EM, Rebeyka IM, Ross DB, Hirji M, et al. (2006). The rapid prototyping of anatomic models in pulmonary atresia. J. Thorac. Cardiovasc. Surg. 132: 264-269.

Noecker AM, Chen JF, Zhou Q, White RD, et al. (2006). Development of patient-specific three-dimensional pediatric cardiac models. ASAIO. J. 52: 349-353.

Pentecost JO, Icardo J and Thornburg KL (1999). 3D computer modeling of human cardiogenesis. Comput. Med. Imaging Graph 23: 45-49.

Pentecost JO, Sahn DJ, Thornburg BL, Gharib M, et al. (2001). Graphical and stereolithographic models of the developing human heart lumen. Comput. Med. Imaging Graph 25: 459-463.

Petzold R, Zeilhofer HF and Kalender WA (1999). Rapid prototyping technology in medicine-basics and applications. Comput. Med. Imaging Graph 23: 277-284.

Sailer HF, Haers PE, Zollikofer CP, Warnke T, et al. (1998). The value of stereolithographic models for preoperative diagnosis of craniofacial deformities and planning of surgical corrections. Int. J. Oral Maxillofac. Surg. 27: 327-333.

Schievano S, Migliavacca F, Coats L, Khambadkone S, et al. (2007). Percutaneous pulmonary valve implantation based on rapid prototyping of right ventricular outflow tract and pulmonary trunk from MR data. Radiology 242: 490-497.

Sodian R, Weber S, Markert M, Rassoulian D, et al. (2007). Stereolithographic models for surgical planning in congenital heart surgery. Ann. Thorac. Surg. 83: 1854-1857.

Spence MS, Thomson JD, Weber N and Qureshi SA (2006). Transient renal failure due to hemolysis following transcatheter closure of a muscular VSD using an Amplatzer muscular VSD occluder. Catheter Cardiovasc. Interv. 67: 663-667.

Winder J and Bibb R (2005). Medical rapid prototyping technologies: state of the art and current limitations for application in oral and maxillofacial surgery. J. Oral Maxillofac. Surg. 63:1006-1015. 